\title{
Identifying Complex Causal Dependencies in Configurational Data with Coincidence Analysis
}

by Michael Baumgartner and Alrik Thiem

\begin{abstract}
We present cna, a package for performing Coincidence Analysis (CNA). CNA is a configurational comparative method for the identification of complex causal dependencies-in particular, causal chains and common cause structures-in configurational data. After a brief introduction to the method's theoretical background and main algorithmic ideas, we demonstrate the use of the package by means of an artificial and a real-life data set. Moreover, we outline planned enhancements of the package that will further increase its applicability.
\end{abstract}

\section{Introduction}

Configurational comparative methods (CCMs) subsume techniques for the identification of complex causal dependencies in configurational data using the theoretical framework of Boolean algebra and its various extensions (Rihoux and Ragin, 2009). For example, Qualitative Comparative Analysis (QCA; Ragin, 1987, 2000, 2008)—hitherto the most prominent representative of CCMs-has been applied in areas as diverse as business administration (e.g., Chung, 2001), environmental science (van Vliet et al., 2013), evaluation (Cragun et al., 2014), political science (Thiem, 2011), public health (Longest and Thoits, 2012) and sociology (Crowley, 2013). Besides three stand-alone programs based on graphical user interfaces, three R packages for QCA are currently available, each with a different scope of functionality: QCA (Dusa and Thiem, 2014; Thiem and Duşa, 2013a,c), QCA3 (Huang, 2014) and SetMethods (Quaranta, 2013; an add-on package to Schneider and Wagemann, 2012).

A novel technique called Coincidence Analysis (CNA) has recently joined the family of CCMs (Baumgartner, 2009a,b, 2013a). Like QCA, CNA searches for rigorously minimized sufficient and necessary conditions of causally modeled outcomes, and it implements the same regularity theory of causation as QCA, that is, the theory most prominently advanced by Mackie (1974). Contrary to QCA, however, CNA can treat any number of factors in a processed data set as endogenous (outcomes), and it does not eliminate redundancies from sufficient and necessary conditions by means of QuineMcCluskey optimization (Quine, 1959; McCluskey, 1965), but by means of an optimization algorithm that is custom-built for causal modeling. As a direct consequence of these differences, CNA can identify common cause and causal chain structures. Moreover, the algorithm does not need to be told which factors are endogenous and which ones exogenous; it can infer that from the data. What is more, limited data diversity does not force CNA to resort to counterfactual additions to the data. Finally, while the QCA programs fs/QCA (Ragin and Davey, 2014), fuzzy (Longest and Vaisey, 2008), Tosmana (Cronqvist, 2011) and Kirq (Reichert and Rubinson, 2014) often fail to find all data-fitting models (cf. Baumgartner and Thiem, 2014; Thiem, 2014c; Thiem and Duşa, 2013b), the R implementation of CNA presented in this paper (cna; Ambuehl et al., 2015) not only ensures—as does QCA-that all single-outcome models are identified but additionally recovers the whole space of multiple-outcome models that fit the data.

After an introduction to the theoretical and algorithmic background of CNA, we demonstrate the potential of the cna R package by means of an artificial and a real-life data set. In the final section, we outline planned enhancements of cna that will further increase its applicability.

\section{Theoretical and algorithmic background}

CCMs search for causal dependencies as defined by so-called regularity theories of causation, whose development dates back to David Hume (1711-1776) and John Stuart Mill (1806-1873). By implementing techniques of Boolean algebra, modern regularity theories spell out the notion of causal relevance in terms of redundancy-free (minimized) sufficiency and necessity relations among the elements of analyzed sets of factors (Mackie, 1974; Graßhoff and May, 2001; Baumgartner, 2008, 2013b).

The crucial component of the regularity theoretic definiens of causal relevance is the notion of a minimal theory. A minimal theory of a factor $Z$ is a minimally necessary disjunction of minimally sufficient conditions of $Z$. A conjunction $\Phi$ of coincidently instantiated factors, i.e. $Y_{1} * Y_{2} * \ldots * Y_{n}$, is a minimally sufficient condition of $Z$ if, and only if (iff), $\Phi$ is sufficient for $Z(\Phi \rightarrow Z$ ), and there exists no proper part $\Phi^{\prime}$ of $\Phi$ such that $\Phi^{\prime} \rightarrow Z$. A proper part $\Phi^{\prime}$ of $\Phi$ is the result of eliminating at least 
one conjunct from $\Phi$. A disjunction $\Psi$ of minimally sufficient conditions, i.e. $\Phi_{1}+\Phi_{2}+\ldots+\Phi_{n}$, is a minimally necessary condition of $Z$ iff $\Psi$ is necessary for $Z(Z \rightarrow \Psi)$, and there exists no proper part $\Psi^{\prime}$ of $\Psi$ such that $Z \rightarrow \Psi^{\prime}$. A proper part $\Psi^{\prime}$ of $\Psi$ is the result of eliminating at least one disjunct from $\Psi$. Overall, a minimal theory of $Z$ has the following biconditional form: $\Psi \leftrightarrow Z$ (where $\Psi$ is an expression in disjunctive normal form and $Z$ is a single factor).

A minimal theory represents the causally interpretable dependencies of sufficiency and necessity among the factors contained in a data set $\delta$. That is, causal relevance can be defined in terms of membership in a minimal theory, or more specifically: a factor $A$ is causally relevant to a factor $B$ in a data set $\delta$ iff $\delta$ entails a minimal theory $\Psi \leftrightarrow B$ such that $A$ is contained in $\Psi$.

CNA aims to infer minimal theories from $\delta$ by, first, identifying sufficient and necessary conditions in $\delta$ and by, second, minimizing those conditions. To the latter end, CNA tests the redundancy of factors by eliminating them from sufficient and necessary conditions and checking whether the remaining conditions are still sufficient and necessary, respectively. More specifically, to determine whether a sufficient condition $Y_{1} * Y_{2} * \ldots * Y_{h}$ of a factor $Z$ is minimally sufficient, CNA systematically eliminates conjuncts from $Y_{1} * Y_{2} * \ldots * Y_{h}$. For each conjunction that results from such an elimination, say for $Y_{2} * Y_{3} * \ldots * Y_{h}$, CNA then parses the processed data $\delta$ to check whether $\delta$ contains $Y_{2} * Y_{3} * \ldots * Y_{h}$ in combination with the negation of $Z$, i.e. $z$. If $\delta$ does not contain such a configuration, $Y_{2} * Y_{3} * \ldots * Y_{h}$ is itself sufficient for $Z$, which means that $Y_{1}$ is redundant. CNA then proceeds to eliminate the next conjunct from $Y_{2} * Y_{3} * \ldots * Y_{h}$ and tests for further redundancies, until no more redundancies are found. By contrast, if $\delta$ contains the configuration $Y_{2} * Y_{3} * \ldots * Y_{h}$ in combination with $z, Y_{1}$ makes a difference to $Z$ and is, thus, not redundant. Accordingly, CNA re-adds $Y_{1}$ to $Y_{2} * Y_{3} * \ldots * Y_{h}$ and proceeds to eliminate $Y_{2}$, and so forth.

Similarly, to determine whether a complex necessary condition $\Phi_{1}+\Phi_{2}+\ldots+\Phi_{h}$ of a factor $Z$ is minimally necessary, CNA systematically eliminates disjuncts from $\Phi_{1}+\Phi_{2}+\ldots+\Phi_{h}$ and checks for every resulting disjunction, say for $\Phi_{2}+\Phi_{3}+\ldots+\Phi_{h}$, whether it is still necessary for $Z$, i.e. whether $\delta$ contains a configuration featuring $Z$ without any of the disjuncts in $\Phi_{2}+\Phi_{3}+\ldots+\Phi_{h}$. If $\delta$ does not contain such a configuration, $\Phi_{2}+\Phi_{3}+\ldots+\Phi_{h}$ is still necessary for $Z$, which means that the eliminated disjunct $\Phi_{1}$ is redundant. Next, $\Phi_{2}+\Phi_{3}+\ldots+\Phi_{h}$ is tested for further redundancies, until no more redundancies are found.

CNA does not presuppose that certain factors in $\delta$ can be identified as endogenous prior to applying CNA. In principle, CNA is designed to recover and rigorously minimize all relationships of sufficiency and necessity among the factors in $\delta$. In practice, however, it is often known from the outset which factors are exogenous and which endogenous. What is more, often enough theoretical knowledge is available to order the factors in $\delta$ causally, where a causal ordering is a relation $Y_{i} \prec Y_{j}$ entailing that $Y_{j}$ cannot be a cause of $Y_{i}$ (e.g., because $Y_{i}$ is instantiated temporally before $Y_{j}$ ). That is, an ordering excludes certain causal dependencies but does not stipulate any. Accordingly, in addition to a data set $\delta$, CNA may be given a subset W of endogenous factors (i.e. possible effects) in $\delta$ and an ordering $\prec$ over the factors in $\delta$ as input. Minimally sufficient and necessary conditions are then calculated for the members of $W$ in accordance with $\prec$ only.

Recovered minimal theories of the elements of $\mathrm{W}$ are issued as so-called atomic solution formulas. If CNA finds an atomic solution formula $\Psi_{1} \leftrightarrow Z_{i}$ and an atomic solution formula $\Psi_{2} \leftrightarrow Z_{j}$ such that $Z_{i} \neq Z_{j}$ and $\Psi_{1}$ and $\Psi_{2}$ have at least one factor in common or $Z_{i}$ appears in $\Psi_{2}$ or $Z_{j}$ appears in $\Psi_{1}$, then CNA builds the complex solution formula $\left(\Psi_{1} \leftrightarrow Z_{i}\right) *\left(\Psi_{2} \leftrightarrow Z_{j}\right)$. Configurational data regularly underdetermine their own causal modeling, with the effect that multiple atomic and complex solution formulas fit the data equally well. In cases of such model ambiguities, CNA provides an overview over the whole model space by returning all data-fitting solution formulas.

As causally analyzed data tend to be noisy, that is, confounded by uncontrolled (unmeasured) causes of endogenous factors, it often happens that no configuration of factors is strictly sufficient or necessary for a given $Z \in W$. To still extract some (tentative) causal information from such data, Ragin (2006) has introduced so-called consistency and coverage measures (with values between 0 and 1). Consistency reproduces the degree to which the behavior of a given outcome obeys a corresponding sufficiency or necessity relationship (or a whole solution formula), whereas coverage reproduces the degree to which a sufficiency or necessity relationship (or a whole solution formula) accounts for the behavior of the corresponding outcome. If data cannot be causally modeled with maximal consistency and coverage scores, CNA invites its users to gradually lower consistency and coverage thresholds until solution formulas can be built.

The cna package by Ambuehl et al. (2015) implements the methodological protocol of CNA as sketched above. For more details on the background assumptions of CNA, its minimization algorithm, and its relation to other configurational methods such as QCA, we refer interested readers to Baumgartner (2009a). 


\section{Examples}

\section{Hypothetical data}

In the following, we illustrate the main steps in using the cna package. First, we employ a hypothetical data set from Baumgartner (2009a) to investigate the causal dependencies among five factors hypothesized to constitute a causal structure behind the overall level of education in western democratic countries. These five factors are "strong unions" $(U ; 1=$ strong, $0=$ not strong), "high level of disparity" ( $D ; 1=$ high, $0=$ not high), "strong left parties" ( $L ; 1=$ strong, $0=$ not strong), "high gross national product" (GNP; G; $1=$ high, $0=$ not high) and "high level of education" ( $E ; 1=$ high, $0=$ not high). The data for eight countries are presented in Table 1.

\begin{tabular}{llllll}
\hline Case & $U$ & $D$ & $L$ & $G$ & $E$ \\
\hline 1 & 1 & 1 & 1 & 1 & 1 \\
2 & 1 & 1 & 1 & 0 & 1 \\
3 & 1 & 0 & 1 & 1 & 1 \\
4 & 1 & 0 & 1 & 0 & 1 \\
5 & 0 & 1 & 1 & 1 & 1 \\
6 & 0 & 1 & 1 & 0 & 1 \\
7 & 0 & 0 & 0 & 1 & 1 \\
8 & 0 & 0 & 0 & 0 & 0 \\
\hline
\end{tabular}

Table 1: Exemplary data to be analyzed by CNA.

The cna package comes with an integrated bundle of six data sets from various areas of the social sciences. That bundle also includes the data in Table 1 as the data frame d.educate. Accordingly, the first step to causally model Table 1 by means of CNA is to load the cna package along with the d. educate data.

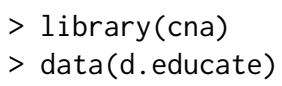

The heart of the cna package is constituted by the $\mathrm{cna}($ ) function. It is the function that identifies and minimizes dependencies of sufficiency and necessity in the data, which can be given to cna() either in terms of a Boolean data frame or of a truth table as produced by the truthTab() function. Essentially, truthTab() simply merges multiple rows of a data frame featuring the same configuration into one row, such that each row of the resulting truth table corresponds to one determinate configuration. The number of occurrences (cases) and an enumeration of the cases are saved as attributes ' $n$ ' and 'cases', respectively. As Table 1 does not contain multiple rows with identical configurations, the application of truthTab() is uncalled for and we can directly pass d. educate on to cna(). Moreover, let us assume that we have no prior causal knowledge about the underlying causal structure, such that we cannot additionally supply a causal ordering. The following is the default output returned by cna().

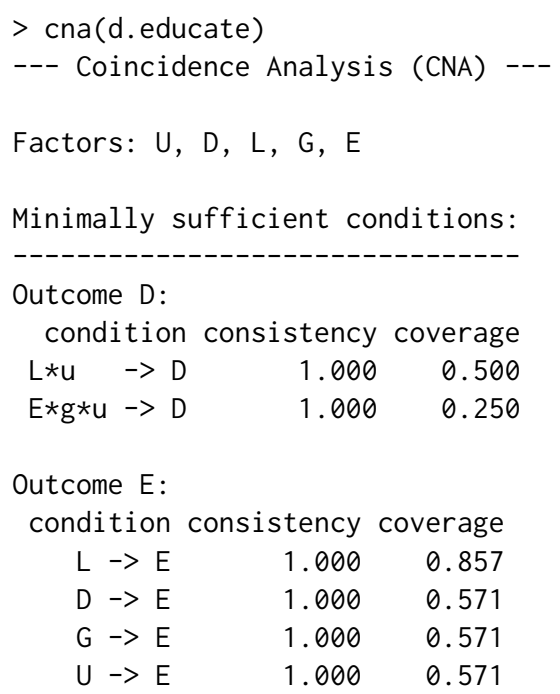




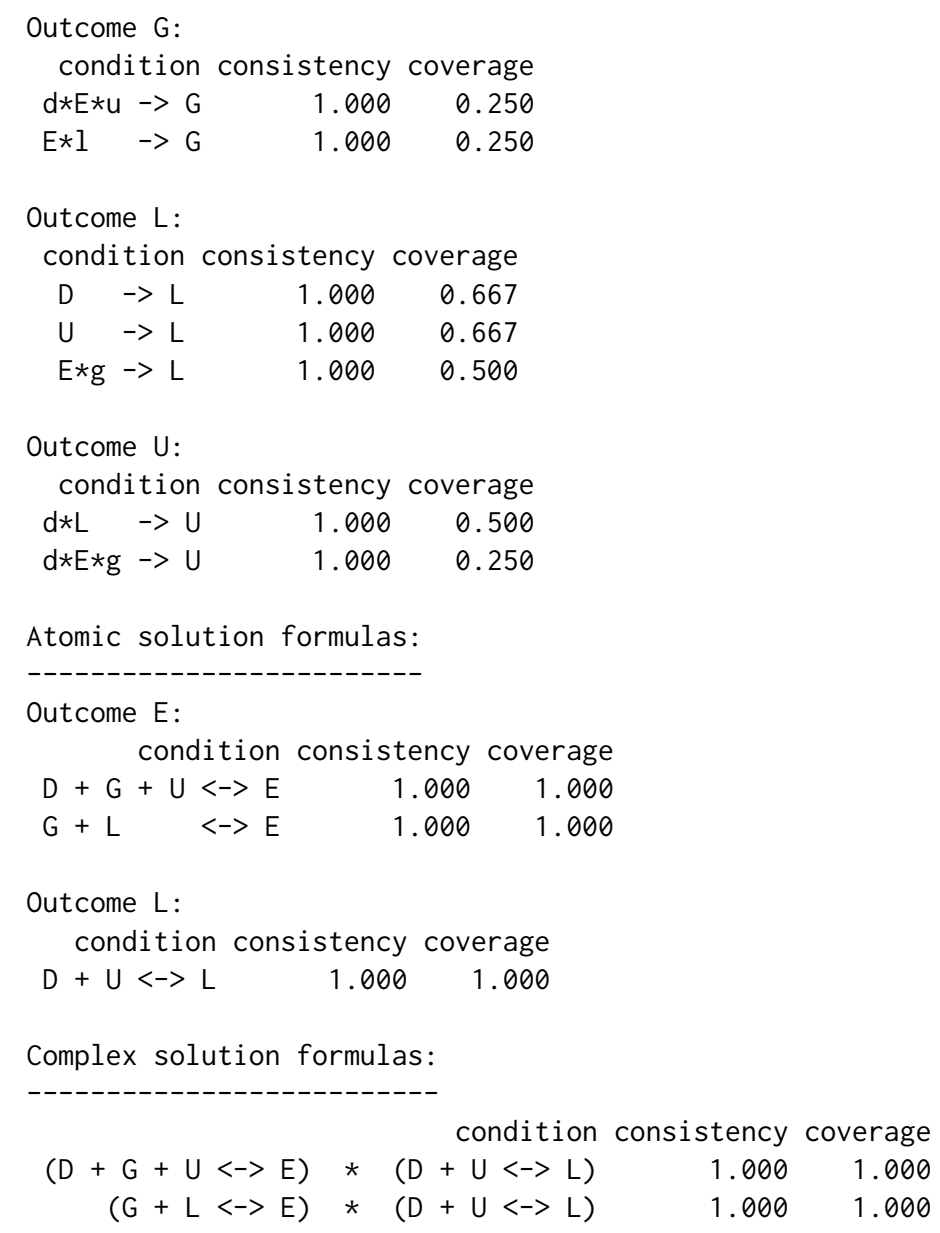

First, cna() lists all minimally sufficient conditions of all factors in d. educate, second, it reports the atomic solution formulas for the factors that can be modeled as endogenous factors, and third, it specifies the resulting complex solutions. All solution types come with corresponding consistency and coverage scores. In case of Table 1, these scores reach maximal values for both atomic and complex solution formulas. Thus, the d. educate data are as good as configurational data can possibly get.

The above results show that the causal structure generating Table 1 features two endogenous factors, viz. "strong left parties" $(L)$ and "high level of education" $(E)$. Moreover, there is one atomic solution for $L$ and there are two for $E$. Overall, cna() infers that the d. educate data can be modeled in terms of the two complex structures depicted in Figure 1. Graph 1a represents a common cause structure, in which "high level of disparity" $(D)$ and "strong unions" $(U)$ appear as direct common causes of $L$ and $E$, whereas Graph 1 b depicts a causal chain such that $D$ and $U$ are direct causes of $L$, which in turn is a direct cause of $E$. As the data in Table 1 are optimal by all standards of configurational modeling, there is no way to determine which of these two structures is the true or correct one.

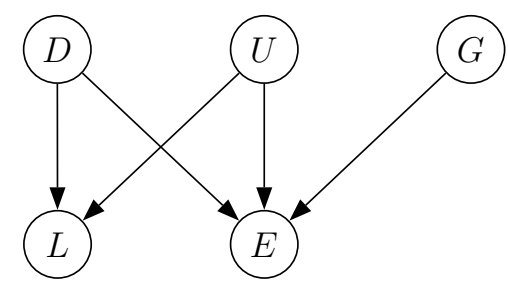

(a) Common cause structure

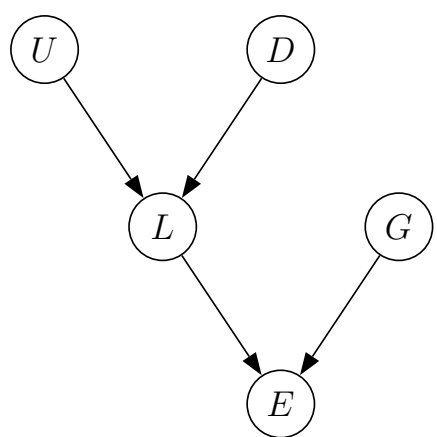

(b) Causal chain

Figure 1: Visualization of dependency structures in d. educate data. 


\section{Real-life data}

This subsection illustrates further functionalities of the cna package on the basis of a real-life data set. To this end, we choose the study by Lam and Ostrom (2010), who analyze the effects of an irrigation experiment in the course of development interventions on the Indrawati River watershed in the central hills of Nepal. Among other things, the authors investigate the causal relevance of five exogenous factors on "persistent improvement in water adequacy at the tail end in winter" $(W)$, which takes the value 1 when farmers at the tail end of the watershed persistently receive the water they need in winter, and the value 0 otherwise. The five exogenous factors are: "continual assistance on infrastructure improvement" $(A)$, "existence of a set of formal rules for irrigation operation and maintenance" $(R)$, "existence of provisions of fines" $(F)$, “existence of consistent leadership" $(L)$, and "existence of collective action among farmers for system maintenance" $(C)$, for all of which the values 1 and 0 represent "yes" and "no", respectively. The relevant data set, which comprises 15 cases, is included in the cna package as the data frame d.irrigate.

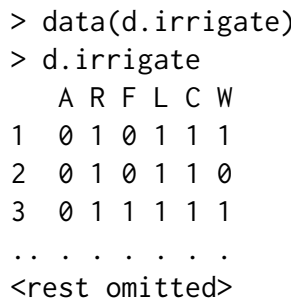

Lam and Ostrom (2010) assume that $W$ is the ultimate outcome of the data-generating causal structure. This background assumption can be given to cna() by means of the argument ordering, which takes a list of character vectors referring to the factors in the data frame as input. In case of $d$.irrigate, the intended ordering is this: ordering = list (c ("A", "R", " $F$ ", " $L "$, " $C$ "), "W"). It determines that $W$ is causally located after $A, R, F, L, C$, meaning that the former cannot be a cause of the latter. Moreover, as this data frame does not comprise all relevant factors for $W$, it is no longer possible to reach perfect coverage scores. In the following analysis, we set the coverage threshold (cov) to 0.9 and extract only the complex solution formulas from the resulting solution object via the function $\operatorname{csf}()$.

$>$ sol1 <- cna(d.irrigate, ordering = list (c("A", "R", "F", "L", "C"), "W"), cov = 0.9) $>\operatorname{csf}(\operatorname{sol} 1)$

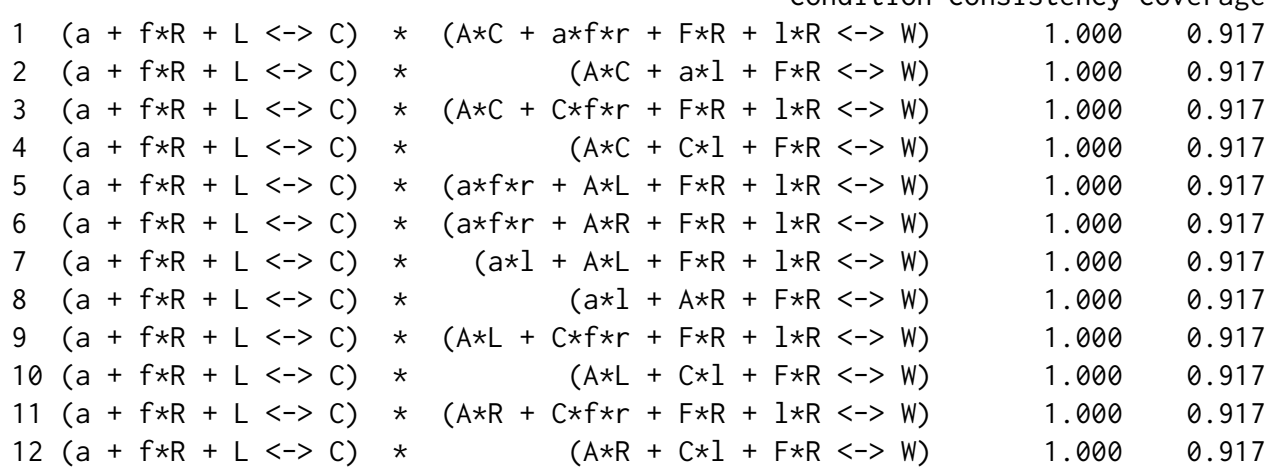

This output of cna() shows that not only $W$ can be modeled as an endogenous factor, but also C-a fact which is overlooked by Lam and Ostrom (2010) due to their reliance on QCA with its focus on single-outcome structures. cna() returns one atomic solution formula for $C$ and 12 for $W$, yielding a total of 12 complex solution formulas that fare equally well with respect to all parameters of model fit. According to some of these models, the behavior of $C$ and $W$ is regulated by a common cause structure; according to others, the underlying structure is a causal chain.

To generate models for negative outcomes, $\mathrm{cna}($ ) provides the argument notcols, which takes a character vector of factors to be negated as input. In the following analysis, we set cov to 0.66 and negate the factors $C$ and $W$ (which then must also appear negatively in the ordering). Moreover, we pass the solution object on to the print () function, which provides arguments determining the number of solutions to print (nsolutions) and what elements of the solution to print (what). The what argument takes a character vector as input, where " $t$ " prints the truth table, " $m$ " the minimally sufficient conditions, "a" the atomic solution formulas, "c" the complex solution formulas, and "all" returns all solution elements.

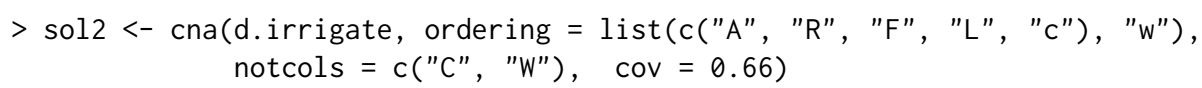


$>\operatorname{print}\left(\right.$ sol2, nsolutions $=3$, what $=$ "a, $\left.c^{\prime \prime}\right)$

--- Coincidence Analysis (CNA) ---

Causal ordering:

$A, R, F, L, C<w$

Atomic solution formulas:

$\begin{array}{rrrr}\text { Outcome } R: & & & \\ & \text { condition } & \text { consistency } & \text { coverage } \\ A * C+f * L & <->R & 1.000 & 0.667 \\ A * F+f * L & <->R & 1.000 & 0.667 \\ A * L+f * L+F * I \stackrel{->R}{R} & 1.000 & 0.667\end{array}$

Outcome w:

condition consistency coverage

$\begin{array}{lrr}A * r+F * r<->w & 1.000 & 0.667 \\ A * r+L * r<->w & 1.000 & 0.667 \\ C * f+F * r<->w & 1.000 & 0.667 \\ \ldots \text { (total no. of formulas: } & \text { 6) }\end{array}$

Complex solution formulas:

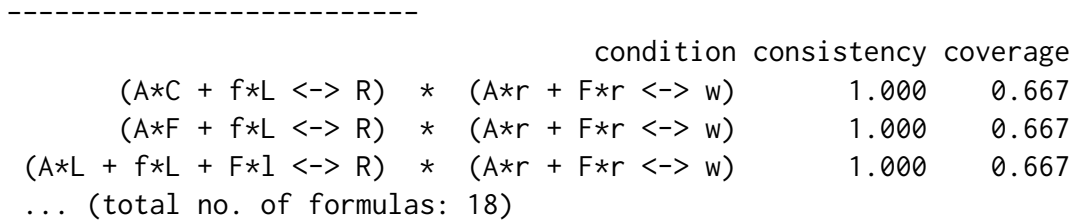

Finally, the condition() function provides assistance to inspect the properties of sufficient and necessary conditions in a data frame, most notably, of minimally sufficient and necessary conditions that appear in solution formulas returned by $c n a()$. It takes a vector of strings specifying Boolean functions as input, reveals which configurations and cases instantiate a given condition or solution, and lists consistency, coverage, as well as unique coverage scores (cf. Ragin, 2008, 63-68). Below, we investigate the properties of the first atomic solution for outcome $w$ from the previous analysis.

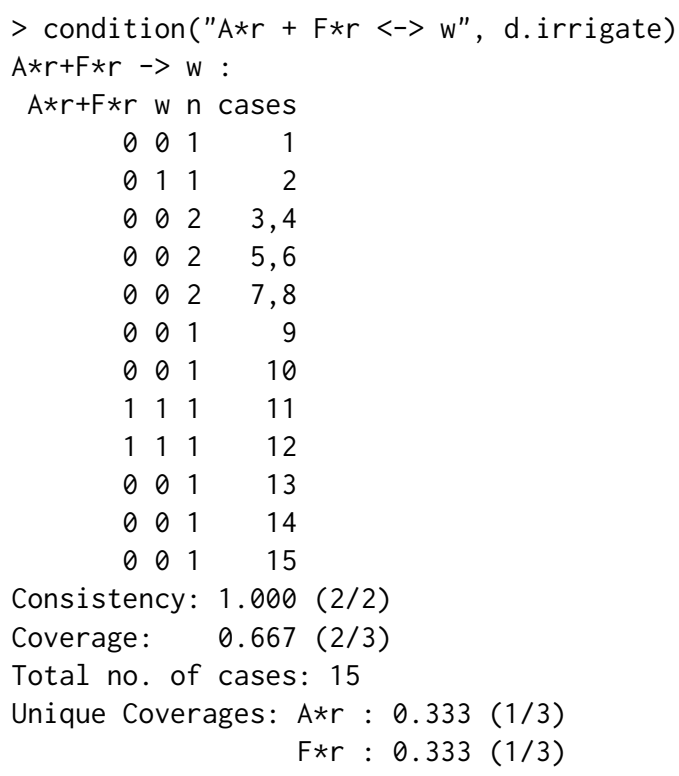

The first two columns of the table returned by condition() indicate the configurations instantiating (' 1 ') and not instantiating $\left({ }^{\prime} O^{\prime}\right)$ the disjunction $A * r+F * r$ and the outcome $w$, respectively. The third column specifies how many cases in the associated data feature a corresponding configuration, and the forth column lists these cases. According to the above output, hence, $A * r+F * r$ covers the instances of $w$ in cases 11 and 12 and leaves the occurrence of $w$ in case 2 uncovered. Consequently, the overall solution coverage is $2 / 3$, with each disjunct uniquely covering one of the instances of $w$. 


\section{Summary and outlook}

We have presented cna, an R package implementing Coincidence Analysis (CNA), which is a method for the identification of multi-outcome structures in configurational data. CNA not only differs from QCA — the standard method of configurational causal modeling-by relaxing the single-outcome restriction but also by not drawing on Quine-McCluskey optimization for the elimination of redundancies from sufficient and necessary conditions. Instead, CNA employs its own minimization algorithm that is custom-built for causal modeling purposes.

At this stage of development, cna still requires bivalent variables. Planned future enhancements include the capability to process multivalent factors that generate crisp sets (Thiem, 2013) and bivalent factors with fuzzy sets (Smithson and Verkuilen, 2006). Possibilities to merge these constructs in multivalent factors with fuzzy sets, as has recently been suggested in the context of QCA (Thiem, 2014a), will be explored as well. In this connection, aspects of alternative procedures proposed in the context of minimization with fuzzy sets may be incorporated where appropriate (Eliason and Stryker, 2009). Finally, functionality for sensitivity diagnostics that facilitates robustness tests is envisaged (Thiem, 2014b).

Complex causal structures are communicated most effectively to readers of scientific articles in the form of graphs rather than formulas. This is all the more true for multivalent factors. In this regard, functionality that translates cna solutions into corresponding graphs enjoys high priority on the list of future enhancements.

\section{Acknowledgment}

This work was generously supported by the Swiss National Science Foundation, grant number PP00P1_144736.

\section{Bibliography}

M. Ambuehl, M. Baumgartner, R. Epple, A. Kauffmann, and A. Thiem. cna: A Package for Coincidence Analysis (CNA), 2015. URL http://CRAN. R-project.org/package=cna. R Package Version 1.0-3. [p176, 177]

M. Baumgartner. Regularity theories reassessed. Philosophia, 36(3):327-354, 2008. [p176]

M. Baumgartner. Inferring causal complexity. Sociological Methods E Research, 38(1):71-101, 2009a. [p176, 177, 178]

M. Baumgartner. Uncovering deterministic causal structures: A Boolean approach. Synthese, 170(1): 71-96, 2009b. [p176]

M. Baumgartner. Detecting causal chains in small-n data. Field Methods, 25(1):3-24, 2013a. [p176]

M. Baumgartner. A regularity theoretic approach to actual causation. Erkenntnis, 78:85-109, 2013b. [p176]

M. Baumgartner and A. Thiem. When there is more than meets the eye: Model ambiguities in configurational comparative research. Paper presented at the 2nd International QCA Expert Workshop, ETH Zürich, 2014. [p176]

C.-N. Chung. Markets, culture and institutions: The emergence of large business groups in Taiwan, 1950s-1970s. Journal of Management Studies, 38(5):719-745, 2001. [p176]

D. Cragun, R. D. DeBate, S. T. Vadaparampil, J. Baldwin, H. Hampel, and T. Pal. Comparing universal Lynch syndrome tumor-screening programs to evaluate associations between implementation strategies and patient follow-through. Genetics in Medicine, 16(10):773-782, 2014. [p176]

L. Cronqvist. Tosmana: Tool for Small-n Analysis, Version 1.3.2.0 [computer program]. University of Trier, Trier, 2011. [p176]

M. Crowley. Gender, the labor process and dignity at work. Social Forces, 91(4):1209-1238, 2013. [p176]

A. Dusa and A. Thiem. Qualitative Comparative Analysis, 2014. URL http://CRAN. R-project.org/ package=QCA. R Package Version 1.1-4. [p176]

S. R. Eliason and R. Stryker. Goodness-of-fit tests and descriptive measures in fuzzy-set analysis. Sociological Methods \& Research, 38(1):102-146, 2009. [p182] 
G. Graßhoff and M. May. Causal regularities. In W. Spohn, M. Ledwig, and M. Esfeld, editors, Current issues in causation, pages 85-114. Mentis, Paderborn, 2001. [p176]

R. Huang. QCA3: Yet Another Package for Qualitative Comparative Analysis, 2014. URL http://CRAN. Rproject. org/package $=Q C A 3$. R package version 0.0-7. [p176]

W. F. Lam and E. Ostrom. Analyzing the dynamic complexity of development interventions: Lessons from an irrigation experiment in Nepal. Policy Sciences, 43(1):1-25, 2010. [p180]

K. C. Longest and P. A. Thoits. Gender, the stress process, and health: A configurational approach. Society and Mental Health, 2(3):187-206, 2012. [p176]

K. C. Longest and S. Vaisey. fuzzy: A program for performing Qualitative Comparative Analyses (QCA) in Stata. Stata Journal, 8(1):79-104, 2008. [p176]

J. L. Mackie. The Cement of the Universe: A Study of Causation. Clarendon Press, Oxford, 1974. [p176]

E. J. McCluskey. Introduction to the Theory of Wwitching Circuits. Princeton University Press, Princeton, 1965. [p176]

M. Quaranta. SetMethods: A package companion to "Set-theoretic methods for the social sciences", 2013. URL http://CRAN.R-project.org/package=SetMethods. R package version 1.0. [p176]

W. v. O. Quine. On cores and prime implicants of truth functions. The American Mathematical Monthly, 66(9):755-760, 1959. [p176]

C. C. Ragin. The Comparative Method: Moving Beyond Qualitative and Quantitative Strategies. University of California Press, Berkeley, 1987. [p176]

C. C. Ragin. Fuzzy-Set Social Science. University of Chicago Press, Chicago, 2000. [p176]

C. C. Ragin. Set relations in social research: Evaluating their consistency and coverage. Political Analysis, 14(3):291-310, 2006. [p177]

C. C. Ragin. Redesigning Social Inquiry: Fuzzy Sets and Beyond. University of Chicago Press, Chicago, 2008. [p176, 181]

C. C. Ragin and S. Davey. fs/QCA: Fuzzy-set/Qualitative Comparative Analysis, Version 2.5 [computer program]. Department of Sociology, University of California, Irvine, 2014. [p176]

C. Reichert and C. Rubinson. Kirq, Version 2.1.12 [computer program]. University of Houston-Downtown, Houston, 2014. [p176]

B. Rihoux and C. C. Ragin, editors. Configurational Comparative Methods: Qualitative Comparative Analysis (QCA) and Related Techniques. Sage, London, 2009. [p176]

C. Q. Schneider and C. Wagemann. Set-Theoretic Methods for the Social Sciences: A Guide to Qualitative Comparative Analysis (QCA). Cambridge University Press, Cambridge, 2012. [p176]

M. Smithson and J. Verkuilen. Fuzzy Set Theory: Applications in the Social Sciences. Sage, London, 2006. [p182]

A. Thiem. Conditions of intergovernmental armaments cooperation in Western Europe, 1996-2006. European Political Science Review, 3(1):1-33, 2011. [p176]

A. Thiem. Clearly crisp, and not fuzzy: A reassessment of the (putative) pitfalls of multi-value QCA. Field Methods, 25(2):197-207, 2013. [p182]

A. Thiem. Unifying configurational comparative methods: Generalized-set Qualitative Comparative Analysis. Sociological Methods E Research, 43(2):313-337, 2014a. [p182]

A. Thiem. Membership function sensitivity of descriptive statistics in fuzzy-set relations. International Journal of Social Research Methodology, 17(6):625-642, 2014b. [p182]

A. Thiem. Navigating the complexities of Qualitative Comparative Analysis: Case numbers, necessity relations, and model ambiguities. Evaluation Review, 38(6):487-513, 2014c. [p176]

A. Thiem and A. Duşa. QCA: A package for Qualitative Comparative Analysis. The R Journal, 5(1): 87-97, 2013a. [p176] 
A. Thiem and A. Duşa. Boolean minimization in social science research: A review of current software for Qualitative Comparative Analysis (QCA). Social Science Computer Review, 31(4):505-521, 2013b. [p176]

A. Thiem and A. Duşa. Qualitative Comparative Analysis with R: A User's Guide. Springer, New York, 2013c. [p176]

N. van Vliet, A. Reenberg, and L. V. Rasmussen. Scientific documentation of crop land changes in the Sahel: A half empty box of knowledge to support policy? Journal of Arid Environments, 95:1-13, 2013. [p176]

Michael Baumgartner

Department of Philosophy

University of Geneva

Rue de Candolle 2 / Bât. Landolt

1211 Geneva

Switzerland

michael. baumgartner@unige.ch

Alrik Thiem

Department of Philosophy

University of Geneva

Rue de Candolle 2 / Bât. Landolt

1211 Geneva

Switzerland

alrik.thiem@unige.ch 